\title{
Pathogen taxonomy updates at the Comprehensive Antibiotic Resistance Database: Implications for molecular epidemiology
}

\author{
Kara K. Tsang, David J. Speicher, and Andrew G. McArthur*
}

M.G. DeGroote Institute for Infectious Disease Research, Department of Biochemistry and Biomedical Sciences, DeGroote School of Medicine, McMaster University, Hamilton, Ontario L8S 4K1, Canada

*To whom correspondence should be addressed.

Contact: mcarthua@mcmaster.ca

\begin{abstract}
With the increasing use of genome sequencing as a surveillance tool for molecular epidemiology of antimicrobial resistance, we are seeing an increased intersection of genomics, microbiology, and clinical epidemiology. Clear nomenclature for AMR gene families and pathogens is critical for communication. For CARD release version 3.0.3 (July 2019), we updated the entire CARD database to reflect the latest pathogen names. In total, we detected 48 name changes or updates, some of which reflect major changes in familiar names.
\end{abstract}

Keywords: antimicrobial resistance, biocuration, microbial nomenclature, molecular epidemiology

\section{Introduction}

With the increasing use of genome sequencing as a surveillance tool for molecular epidemiology of antimicrobial resistance (AMR) $(1,2)$, we are seeing an increased intersection of genomics, microbiology, and clinical epidemiology. As such, clear nomenclature for AMR gene families and pathogens is critical for communication. At the Comprehensive Antibiotic Resistance Database (CARD) (3), we provide highly curated reference data on the molecular basis of AMR. As a rule, sequences added to CARD must include peer-reviewed, published experimental evidence of antibiotic minimum inhibitory concentration (MIC) elevated above that of a control, plus availability of the DNA sequence of the corresponding gene or mutant in GenBank (4). All data is organized ontologically, most notably using the Antibiotic Resistance Ontology and an ontological mirror of GenBank's Taxonomy Database (3). Yet, since its inception CARD has not performed an update of the microbiological taxonomy and nomenclature underlying its organization of AMR sequences, predicted allelic variants, resistome annotations, and epidemiological estimates. As such, for CARD release version 3.0.3 (July 2019) we added a new software module to our CARD Quality Control tools to detect nomenclatural changes in GenBank not incorporated into CARD, which we then used to update the CARD database to reflect the latest pathogen names. In total, we detected 48 name changes or updates, some of which reflect major changes in familiar names (Table 1). These changes are outlined below with citations for convenience and clarity, using the acronym p.k.a. for "previously known as".

\section{Clostridioides difficile (p.k.a. Clostridium difficile)}

CARD recently (CARD version 3.0.3, July 2019) performed a literature review of genes and mutations conferring AMR to the Gram-positive spore-forming bacterium $C$. difficile, a causative agent of diarrheal disease and colitis via colonization of the human colon. Familiar to clinicians as Clostridium difficile, Yutin \& Galperin (5) proposed a name change in 2013 to Peptoclostridium difficile based on genomic and evolutionary analyses, which proved to be unpopular due to loss of the familiar ' $C$. diff' and 'CDAD' (Clostridium difficile-associated diarrhea) monikers. Subsequent phylogenetic analysis by Lawson et al. (6) confirmed the genus Clostridium had been poorly defined, that $C$. difficile was indeed more closely related to Peptoclostridium than other Clostridium, and supported the new genus Clostridioides (meaning 'Clostridia-like'). This genus contains Clostridium mangenotii and Clostridium difficile as Clostridioides mangenotii and Clostridioides difficile, respectively. This new name conveniently maintains the ' $C$. diff' moniker.

\section{Cutibacterium acnes (p.k.a. Propionibacterium acnes)}

Propionibacterium acnes is a Gram-positive bacterium involved in the pathogenesis of acne. Originally known Bacillus acnes, the species was renamed Cutibacterium acnes by Scholz \& Kilian (7) based on genome and phylogenetic analysis. In CARD, Cutibacterium acnes has been associated with gyrA and $16 \mathrm{~S}$ rRNA mutations conferring resistance to fluoroquinolones and tetracycline, respectively.

\section{Kitasatospora aureofaciens (p.k.a. Streptomyces aureofaciens)}

Streptomyces aureofaciens is widely known as a source of many tetracycline antibiotics (8) and in many ways represents the archetype for antibiotic producing Streptomyces. However, multi-gene phylogenetic analyses (9) have illustrated that this species is a member of the genus Kitasatospora, hence a name change to Kitasatospora aureofaciens has been adopted.

\section{Borreliella burgdorferi (p.k.a. Borrelia burgdorferi)}

The spirochete bacteria Borrelia burgdorferi is a tick-borne causative agent of Lyme disease and thus a focus of considerable scrutiny for vaccine and drug development, as well as molecular epidemiology (10). Yet, phylogenomic investigation has split the genus into Borrelia and Borreliella (11), resulting in the new and validated name Borreliella burgdorferi. 
Kara K. Tsang, David J. Speicher, and Andrew G. McArthur 


\section{Klebsiella aerogenes (p.k.a. Enterobacter aerogenes)}

The familiar ESKAPE pathogen Enterobacter aerogenes has a long nomenclatural history. This Gram-negative, opportunistic pathogen was originally included as Aerobacter aerogenes and later renamed Enterobacter aerogenes based on nomenclatural rules, but as early as 1971 it was realized that not all strains of Enterobacter were likely to be of the same genus and that Enterobacter aerogenes was more likely a member of Klebsiella $(\mathbf{1 2}, \mathbf{1 3})$. Nomenclatural rules at the time blocked this name change, but recognition of overlapping types (NCTC 10006, ATCC 13048) resulted in the short-lived moniker Klebsiella mobilis (i.e. Klebsiella mobilis and Enterobacter aerogenes are homotypic synonyms). Tindall et al. (13) reviewed this naming history and current nomenclatural rules, resulting in the definitive and widely adopted change to Klebsiella aerogenes. It is worth noting that this taxonomic revision complicates the familiar ESKAPE pathogens acronym for prevalent, AMR hospital acquired infections: Enterococcus faecium, Staphylococcus aureus, Klebsiella pneumoniae, Acinetobacter baumannii, Pseudomonas aeruginosa, and Enterobacter species ("Klebsiella pneumoniae" could be replaced with "Klebsiella species"). From a phenotypic point of view, care should be taken for assumptions of antimicrobial susceptibilities, since Klebsiella aerogenes has a different AMR gene compliment than other Klebsiella.

\section{Mycobacterium, Mycobacteroides, \& Mycolicibacterium}

Until recently, the diversity of species related to Mycobacterium tuberculosis have been placed in the same genus, e.g. Mycobacterium abscessus, Mycobacterium chelonae, Mycobacterium fortuitum, Mycobacterium goodii, Mycobacterium smegmatis, Mycobacterium bovis. Yet, recent taxonomic revisions based on comparative genomics and evolutionary studies have distributed these species among three genera with separate evolutionary histories (4, 5): Mycobacterium tuberculosis \& Mycobacterium tuberculosis variant bovis; Mycobacteroides abscessus \& Mycobacteroides chelonae; Mycolicibacterium fortuitum, Mycolicibacterium goodii, \& Mycolicibacterium smegmatis. Other species, not yet included in CARD, have been placed in a fourth \& fifth new genus, Mycolicibacter and Mycolicibacillus (14).

\section{Conclusions}

A large number of databases relating to AMR exist (see CARD's amr_curation repo and Google spreadsheet list of databases at https://github.com/arpcard/amr_curation), yet most curate a molecular sequence once, reflecting its initial report, as is done at CARD. This initial report and entry in GenBank often reflect a gene's original detection in a single pathogen, yet AMR is an evolutionary process involving both evolution of the AMR sequences themselves over time and their horizontal gene transfer among pathogens. As such, AMR databases must do more than form a collection of reference sequences, but over time must track their evolution and epidemiological distribution. To do this well, AMR databases must update molecular sequences as well as our understanding of pathogen relationships. As AMR is an evolutionary phenomena, our analyses of AMR must also have an accurate evolutionary context which is most often reflected in the names of microbes. As illustrated above, bacterial nomenclature is increasingly guided by comparative genomic, phylogenetic, and phylogenomic analyses. Ignoring these nomenclatural changes places our analyses and interpretation of AMR into incorrect context. AMR databases need to update microbial nomenclature to remain relevant. Yet, in clinical settings nomenclatural changes in academia often can appear arbitrary, as familiar monikers are important for clinical diagnostics, public health decisions, and clear lines of communication. As outlined in Dr. Audrey Schuetz's online article (15), changes in nomenclature can disrupt clinical processes and have unanticipated costs. As such, and following GenBank's lead, CARD now strives to follow the latest changes in bacterial nomenclature and as it is based on both the Chado data schema and organized exclusively using ontologies (3), is able to rapidly update names and descriptions within CARD to reflect the latest nomenclature while simultaneously supporting depreciated names as ontological synonyms, allowing users to search CARD using familiar monikers in case they are unfamiliar with recent name changes, allowing them to find the data they need.

\section{Acknowledgements}

This research was funded by the Canadian Institutes of Health Research (PJT-156214) to A.G.M., who also holds a Cisco Research Chair in Bioinformatics, supported by Cisco Systems Canada, Inc. K.K.T. was supported by an Ontario Graduate Scholarship and McMaster University's MacDATA Institute Graduate Fellowship, Michael G. DeGroote Institute for Infectious Disease Research Michael Kamin Hart Memorial Scholarship, Faculty of Health Sciences Graduate Programs Excellence Award, and Biochemistry \& Biomedical Sciences Fred and Helen Knight Enrichment Award. D.J.S. was supported by the Michael G. DeGroote Initiative for Innovation in Healthcare. Computer resources were supplied by the McMaster Service Lab and Repository computing cluster, funded in part by grants to A.G.M. from the Canadian Foundation for Innovation (34531).

\section{References}

1. McArthur AG, Tsang KK. 2017. Antimicrobial resistance surveillance in the genomic age. Ann N Y Acad Sci 1388:7891.

2. McArthur AG, Wright GD. 2015. Bioinformatics of antimicrobial resistance in the age of molecular epidemiology. Curr Opin Microbiol 27:45-50.

3. Jia B, Raphenya AR, Alcock B, Waglechner N, Guo P, Tsang KK, Lago BA, Dave BM, Pereira S, Sharma AN, Doshi S, Courtot M, Lo R, Williams LE, Frye JG, Elsayegh T, Sardar D, Westman EL, Pawlowski AC, Johnson TA, Brinkman FSL, Wright GD, McArthur AG. 2017. CARD 2017: expansion and model-centric curation of the comprehensive antibiotic resistance database. Nucleic Acids Res 45:D566-D573.

4. Benson DA, Cavanaugh M, Clark K, Karsch-Mizrachi I Lipman DJ, Ostell J, Sayers EW. 2013. GenBank. Nucleic Acids Res 41:D36-42.

5. Yutin N, Galperin MY. 2013. A genomic update on clostridial phylogeny: gram-negative spore formers and other misplaced clostridia. Environ Microbiol 15:2631-2641. 
Kara K. Tsang, David J. Speicher, and Andrew G. McArthur

6. Lawson PA, Citron DM, Tyrrell KL, Finegold SM. 2016. Reclassification of Clostridium difficile as Clostridioides difficile (Hall and O'Toole 1935) Prévot 1938. Anaerobe 40:9599.

7. Scholz CFP, Kilian M. 2016. The natural history of cutaneous propionibacteria, and reclassification of selected species within the genus Propionibacterium to the proposed novel genera Acidipropionibacterium gen. nov., Cutibacterium gen. nov. and Pseudopropionibacterium gen. nov. Int J Syst Evol Microbiol 66:4422-4432.

8. DARKEN MA, BERENSON H, SHIRK RJ, SJOLANDER NO. 1960. Production of tetracycline by Streptomyces aureofaciens in synthetic media. Appl Microbiol 8:46-51.

9. Labeda DP, Dunlap CA, Rong X, Huang Y, Doroghazi JR, Ju K-S, Metcalf WW. 2017. Phylogenetic relationships in the family Streptomycetaceae using multi-locus sequence analysis. Antonie Van Leeuwenhoek, 2nd ed. 110:563-583.

10. Tilly K, Rosa PA, Stewart PE. 2008. Biology of infection with Borrelia burgdorferi. Infect Dis Clin North Am 22:21734- v.

11. Adeolu M, Gupta RS. 2014. A phylogenomic and molecular marker based proposal for the division of the genus Borrelia into two genera: the emended genus Borrelia containing only the members of the relapsing fever Borrelia, and the genus Borreliella gen. nov. containing the members of the Lyme disease Borrelia (Borrelia burgdorferi sensu lato complex). Antonie Van Leeuwenhoek, 2nd ed. 105:1049-1072.

12. Bascomb S, Lapage SP, Willcox WR, Curtis MA. 1971. Numerical classification of the tribe Klebsielleae. J Gen Microbiol 66:279-295.

13. Tindall BJ, Sutton G, Garrity GM. 2017. Enterobacter aerogenes Hormaeche and Edwards 1960 (Approved Lists 1980) and Klebsiella mobilis Bascomb et al. 1971 (Approved Lists 1980) share the same nomenclatural type (ATCC 13048) on the Approved Lists and are homotypic synonyms, with consequences for the name Klebsiella mobilis Bascomb et al. 1971 (Approved Lists 1980). Int J Syst Evol Microbiol 67:502-504.

14. Gupta RS, Lo B, Son J. 2018. Phylogenomics and comparative genomic studies robustly support division of the genus Mycobacterium into an emended genus Mycobacterium and four novel genera. Front Microbiol 9:67.

15. Schuetz, A.N. 2017. Celebrating successes and contemplating messes in bacterial taxonomy. https://www.asm.org/Articles/2017/September/Clinical-and-Public-Health-Microbiology-(7) 
Kara K. Tsang, David J. Speicher, and Andrew G. McArthur

Table 1. Taxonomic updates at the Comprehensive Antibiotic Resistance Database, with associated NCBI Taxonomy ID. Bolded taxa are discussed in the text.

\begin{tabular}{|c|c|c|}
\hline Old Name & New Name & NCBI \\
\hline Acinetobacter baumannii ATCC 19606 & Acinetobacter baumannii ATCC $19606=\mathrm{CIP} 70.34=\mathrm{JCM} 6841$ & 575584 \\
\hline Acinetobacter $\mathrm{sp}$. ADP1 & Acinetobacter baylyi ADP1 & 62977 \\
\hline Acinetobacter genomosp. 14 & Acinetobacter colistiniresistens & 70345 \\
\hline Acinetobacter genomosp. 17BJ & Acinetobacter dispersus & 70348 \\
\hline Acinetobacter genomosp. 15 & Acinetobacter variabilis & 70346 \\
\hline Streptomyces caeruleus & Actinoalloteichus cyanogriseus & 195949 \\
\hline Agrobacterium tumefaciens str. C58 & Agrobacterium fabrum str. C58 & 176299 \\
\hline Bacillus amyloliquefaciens subsp. plantarum str. FZB42 & Bacillus velezensis FZB42 & 326423 \\
\hline Borrelia burgdorferi & Borreliella burgdorferi & 139 \\
\hline Borrelia burgdorferi B31 & Borreliella burgdorferi B31 & 224326 \\
\hline Campylobacter jejuni subsp. jejuni NCTC 11168 & Campylobacter jejuni subsp. jejuni NCTC $11168=$ ATCC 700819 & 192222 \\
\hline Epilithonimonas sp. Stok-2 & Chryseobacterium sp. Stok-2 & 1620219 \\
\hline Clostridium difficile & Clostridioides difficile & 1496 \\
\hline Clostridium difficile 630 & Clostridioides difficile 630 & 272563 \\
\hline Propionibacterium acnes & Cutibacterium acnes & 1747 \\
\hline Enterococcus faecalis $\mathrm{HH} 22$ & Enterococcus faecalis EnGen0297 & 491075 \\
\hline Streptomyces aureofaciens & Kitasatospora aureofaciens & 1894 \\
\hline Enterobacter aerogenes & Klebsiella aerogenes & 548 \\
\hline Mycobacterium bovis & Mycobacterium tuberculosis variant bovis & 1765 \\
\hline Mycobacterium bovis AF2122/97 & Mycobacterium tuberculosis variant bovis AF2122/97 & 233413 \\
\hline Mycobacterium bovis BCG str. Mexico & Mycobacterium tuberculosis variant bovis BCG str. Mexico & 717522 \\
\hline Mycobacterium bovis BCG str. Pasteur 1173P2 & Mycobacterium tuberculosis variant bovis BCG str. Pasteur 1173P2 & 410289 \\
\hline Mycobacterium abscessus & Mycobacteroides abscessus & 36809 \\
\hline Mycobacterium chelonae & Mycobacteroides chelonae & 1774 \\
\hline Mycobacterium fortuitum & Mycolicibacterium fortuitum & 1766 \\
\hline Mycobacterium goodii & Mycolicibacterium goodii & 134601 \\
\hline Mycobacterium smegmatis & Mycolicibacterium smegmatis & 1772 \\
\hline Mycobacterium smegmatis str. MC2 155 & Mycolicibacterium smegmatis MC2 155 & 246196 \\
\hline Rhodococcus equi & Rhodococcus hoagii & 43767 \\
\hline Salmonella enterica subsp. enterica serovar Arizona & Salmonella enterica subsp. arizonae & 486989 \\
\hline Salmonella enterica subsp. arizonae str. ATCC BAA-1577 & $\begin{array}{l}\text { Salmonella enterica subsp. arizonae serovar } 41: z 4, z 23:- \text { str. ATCC } \\
\text { BAA- } 1577\end{array}$ & 523833 \\
\hline Salmonella enterica subsp. arizonae serovar $62: \mathrm{z} 4, \mathrm{z} 23:--$ & Salmonella enterica subsp. arizonae serovar 62:z4,z23:- & 41514 \\
\hline Salmonella enterica IIIb 50:k:z & Salmonella enterica subsp. diarizonae serovar 50:k:z & 41512 \\
\hline Salmonella enterica subsp. enterica serovar 4,12;I,- & Salmonella enterica subsp. enterica serovar 4,12:i:- & 353569 \\
\hline Salmonella enterica subsp. enterica serovar Amesterdam & Salmonella enterica subsp. enterica serovar Amsterdam & 593904 \\
\hline $\begin{array}{l}\text { Salmonella enterica subsp. enterica serovar Typhimurium var. } \\
\text { Copenhagen }\end{array}$ & Salmonella enterica subsp. enterica serovar Copenhagen & 353544 \\
\hline $\begin{array}{l}\text { Salmonella enterica subsp. enterica serovar Paratyphi C strain } \\
\text { RKS4594 }\end{array}$ & $\begin{array}{l}\text { Salmonella enterica subsp. enterica serovar Paratyphi C str. } \\
\text { RKS4594 }\end{array}$ & 476213 \\
\hline Salmonella typhimurium DT104 & $\begin{array}{l}\text { Salmonella enterica subsp. enterica serovar Typhimurium str. } \\
\text { DT104 }\end{array}$ & 85569 \\
\hline Salmonella typhimurium SL1344 & $\begin{array}{l}\text { Salmonella enterica subsp. enterica serovar Typhimurium str. } \\
\text { SL1344 }\end{array}$ & 216597 \\
\hline Salmonella enterica VI 1,6,14,25:a:e,n,x & Salmonella enterica subsp. indica serovar $1,6,14,25: \mathrm{a}: \mathrm{e}, \mathrm{n}, \mathrm{x}$ & 59246 \\
\hline Salmonella enterica VII 1,40:g,z51:-- & Salmonella enterica subsp. VII serovar 1,40:g,z51:-- & 41520 \\
\hline Salmonella enterica VII 40:z4,z24:-- & Salmonella enterica subsp. VII serovar 40:z4,z24:-- & 41521 \\
\hline Enterobacteria phage Fels-2 & Salmonella virus Fels2 & 194701 \\
\hline Staphylococcus aureus ED98 & Staphylococcus aureus subsp. aureus ED98 & 665030 \\
\hline Staphylococcus aureus RN4220 & Staphylococcus aureus subsp. aureus RN4220 & 561307 \\
\hline Streptomyces roseosporus NRRL 11379 & Streptomyces filamentosus NRRL 11379 & 457430 \\
\hline Streptomyces roseochromogenes subsp. oscitans & Streptomyces roseochromogenus subsp. oscitans & 149682 \\
\hline Streptomyces roseochromogenes subsp. oscitans DS 12.976 & Streptomyces roseochromogenus subsp. oscitans DS 12.976 & 1352936 \\
\hline
\end{tabular}

